\title{
Noise Reduction in Hyperspectral Images through Spectral Unmixing
}

\author{
Daniele Cerra, Rupert Müller, and Peter Reinartz
}

\begin{abstract}
Spectral unmixing and denoising of hyperspectral images have always been regarded as separate problems. By considering the physical properties of a mixed spectrum, this paper introduces Unmixing-based Denoising, a supervised methodology representing any pixel as a linear combination of reference spectra in a hyperspectral scene. Such spectra are related to some classes of interest, and exhibit negligible noise influences, as they are averaged over areas for which ground truth is available. After the unmixing process, the residual vector is mostly composed by the contributions of uninteresting materials, unwanted atmospheric influences and sensor-induced noise, and is thus ignored in the reconstruction of each spectrum. The proposed method, in spite of its simplicity, is able to remove noise effectively for spectral bands with both low and high Signal-toNoise Ratio. Experiments show that this method could be used to retrieve spectral information from corrupted bands, such as the ones placed at the edge between Ultraviolet and visible light frequencies, which are usually discarded in practical applications. The proposed method achieves better results in terms of visual quality in comparison to competitors, if the Mean Squared Error is kept constant: this leads to question the validity of Mean Squared Error as a predictor for image quality in remote sensing applications.
\end{abstract}

Index Terms-Spectral unmixing, denoising, image restoration, hyperspectral images, mean squared error.

\section{INTRODUCTION}

$\mathbf{T}$ HE spectral range characterizing data acquired by stateof-the-art hyperspectral sensors mostly spans the frequencies between $400 \mathrm{~nm}$ and $2500 \mathrm{~nm}$. Some sensors, such as the Airborne Visible Infrared Imaging Spectrometer (AVIRIS) and the future HySpiri mission, acquire data also in the portion of the spectrum which is placed at the edge between Near Ultraviolet (NUV) frequencies and visible light (380-400 nm) [1]. The spectral bands in this range are affected by noise of different nature which is difficult to model, as it comes from several sources [2]. Such bands are typically characterized by a low Signal to Noise Ratio (SNR), and as a consequence are usually discarded in a preprocessing step common to most practical applications.

For some specific tasks, it would be desirable to keep such spectral information: a typical example is the study of Coloured dissolved organic matter (CDOM) in natural waters. CDOM inhibits phytoplankton productivity by absorbing UV and NUV radiation, affecting in turn remote estimates of clorophyll concentration [3]. As the bands in the NUV-blue portion of the spectrum are usually noisy and therefore difficult to interpret, bands at longer wavelengths characterized by a better SNR are usually preferred to derive empirical indices for its estimation. As an example, in [4] the authors simulate the reflectance at $380 \mathrm{~nm}$ using the bands in the 400-500 $\mathrm{nm}$ portion of the spectrum. Therefore, a specific denoising methodology enabling a direct use of the information in the mentioned spectral range would represent a great aid for such applications.

Denoising is often carried out in image processing through filtering, usually based on convolutions with sliding windows in the image domain, on operations in the frequency domain, or on estimated noise statistics or degradation functions, if these are known for the image acquisition process [5]. In the case of hyperspectral images, the high dimensionality of the data and the correlation between adjacent bands can be exploited to carry out effective denoising procedures based on dimensionality reduction (DR) algorithms, which project the data onto a subspace where meaningful information is preserved, while noise and some high frequencies are discarded [6]. The most well-known DR techniques on top of which denoising algorithms have been defined are the Principal Components Analysis [7] and the Minimum Noise Fraction (MNF) [8]. The latter is often the preferred method to employ as a preprocessing step in hyperspectral data analysis to reduce the data dimensionality, removing at the same time noise and redundancies. The problems arising when using this method have been, on the other hand, seldom addressed. First of all, MNF needs the number of noisy components to be estimated, which is not a trivial problem [9]. In addition, this estimation is different for bands with different SNR, as it is not possible to achieve an optimal denoising for all the bands at the same time. Finally, the usual way of validating the quality of the denoised images in literature is by computing the Mean Squared Error (MSE) between original and reconstructed image, with MNF being optimal in a MSE sense, as it represents each band as a linear combination of other bands minimizing the MSE. This is also the case for other hyperspectral denoising algorithms driven by MSE minimization [10]. Nevertheless, in recent years studies in image processing have shown that degraded images with constant MSE may exhibit very different visual image quality, and indices which capture statistical image properties related to human perception are becoming widely accepted in the image processing community to estimate distortions [11]. Therefore, validation of techniques which are based on MSE minimization and use that same criterion as a quality predictor may be biased.

In [12] the authors explore the correspondences between yet another DR technique, the Independent Components Analysis (ICA), and spectral unmixing [13], which aims at quantitatively decomposing each pixel in signals related to macroscopically pure materials, or endmembers. While the experiments in the aforementioned work are derived from a statistical 
analysis of the data, this paper introduces a supervised denoising algorithm based on spectral unmixing which results from considerations on the physical properties of a mixed pixel, which can be described as follows.

In a first step a set of reference spectra is derived by spatial averaging of neighbouring pixels in each class of interest. Subsequently each image element, considered as a mixed spectrum, is expressed as a linear combination of the reference spectra plus a residual vector, which quantifies the unmixing error. If the considered reference spectra are noise-free and comprise all the relevant classes in the image, such vector will mostly be composed by noise and components of less interest. Therefore, each full spectrum is finally reconstructed ignoring the residual vector. Results obtained through the described Unmixing-based Denoising (UBD) algorithm show a superior visual image quality with respect to the reconstruction using MNF features, if the MSE is kept constant: this could enable in the future new applications exploiting the spectral information contained in bands severely affected by noise. The method works equally well for bands with high SNR, while a different number of components has to be kept in the inverse MNF rotation according to the band SNR. The reported experiments lead to question the validity of MSE as quality predictor in applications to hyperspectral and in general remotely sensed data.

The paper is structured as follows. Section II introduces the proposed denoising methodology based on spectral unmixing. We report experimental results and comparison with MNFbased denoising in section III, and conclude in section IV.

\section{UNMIXING-BASED DENOISING}

A recently described classification methodology for hyperspectral data based on synergetics theory [14] projects any image element onto a "semantic" subspace, in which every dimension represents the similarity to a given class of interest. This procedure inspires a supervised methodology based on spectral unmixing to suppress noise for the bands with low SNR described in the previous section, but that turns out to be effective on any band of a given hyperspectral image.

Given a hyperspectral image element $m$ with $p$ bands, and a training dataset containing $n$ samples from each of $k$ classes, with $k<p$, the Unmixing-based Denoising (UBD) is a simple procedure which can be described as follows. First of all, a set of reference spectra is defined as $A=\left\{x_{1}, \ldots, x_{i}, \ldots, x_{k}\right\}$, where $x_{i}$ is the average of the $n$ spectra belonging to class $i$. Considering the mean value for a given reference spectrum ensures that, if each class is spectrally homogeneous, the presence of noise in it is reduced to a minimum. Such assumptions are also made in [15], in which spectra averaged within some classes of interest are employed to perform a supervised spectral unmixing prior to classification. Even though no assumption on the purity of the reference spectra are made, the image elements belonging to a homogeneous area have a higher prior probability of being related to some pure material [16]. After the selection of the reference spectra, any unmixing procedure can be employed to decompose the signal in a combination of the reference spectra. If we assume this to be linear, we have:

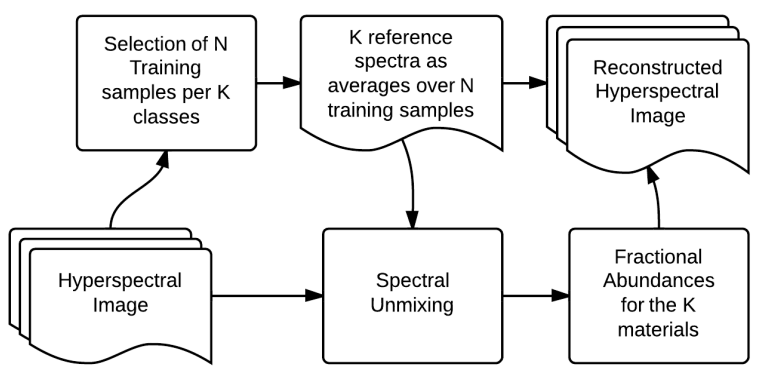

Fig. 1. Workflow for Unmixing-based Denoising. The selected reference spectra are averaged over a given area of interest, which is homogeneous to some degree, and the image is reconstructed as a linear combination of the reference spectra. The residual of the unmixing process is discarded, along with most of the noise in the data.

$$
m=\sum_{i=1}^{k} x_{i} s_{i}+r,
$$

where $s_{i}$ is the fraction or abundance of the reference spectrum $i$ in $m$, and $r$ the residual vector. The latter can be expressed as:

$$
r=r_{1}+r_{2}+r_{n},
$$

where $r_{1}$ is given to components related to materials not present in $A, r_{2}$ is an error given by subtle variations of one or more materials in $A$, and $r_{n}$ is caused by atmospheric influence and instrument-induced noise. The noise here is regarded as additive, as a study in [2] concludes that signal-dependant noise in typical hyperspectral sensors can be neglected. If our classes of interest are well captured in $A$ we are not interested in other materials, and therefore we can ignore $r_{1}$, and if they are homogeneous we expect $r_{n}$ to be predominant over $r_{2}$ for bands with low SNR. Therefore, we can derive a reconstruction $\hat{m}$ for a spectrum $m$ as:

$$
\hat{m}=\sum_{i=1}^{k} x_{i} s_{i},
$$

ignoring $r$, and along with it most of the noise affecting $m$. The workflow is reported in Fig. 1.

The described procedure is based on the assumption that if the contributions to the radiation reflected from a resolution cell are known, the value of noisy bands in that area can be derived by a combination of the average values characterizing each component in that spectral range. The proposed method is supervised and is carried out independently for each pixel. It additionally assumes that the selected spectra in the scene are known or can be reliably estimated, and a certain homogeneity of the classes of interest, which is to be expected in a natural scene rather than in a scene where man-made objects are prevalent.

\section{RESULTS}

\section{A. Salinas dataset}

We analyze an AVIRIS hyperspectral scene containing agricultural fields acquired over the Salinas Valley, USA, of size 
$512 \times 217$ pixels with 192 spectral bands from 0.38 to 2.5 $\mu m$ (water absorption bands removed as in [17]), and with a spatial resolution of $3.7 \mathrm{~m}$. As expected, the band centered at $380 \mathrm{~nm}$ is severely affected by noise(Fig. 2(a)). Ground truth is available for 15 classes in the area reported in Fig. $3(\mathrm{f})$, and we select the average spectrum over a $6 \times 6$ pixels area for each class (the size of the averaging window has been set empirically), resulting in 16 reference spectra (the class corn has been divided in two classes as in [14]). The next step is the choice of the unmixing algorithm for Eq. (1). Recently, interest has arisen in non-linear spectral unmixing methods, but these are not yet mature. We adopt then a standard linear unmixing algorithm based on inversion through Least Squares [13], which do not rely on any noise model which could bias our validation: noise in hyperspectral sensors is often treated as Gaussian, but is instead Poisson distributed and therefore more difficult to model correctly [2]. In the specific, we consider Non-negative Least Squares (NNLS), which has the advantage of being physically meaningful, as in its solution all abundancies are positive. It is also of interest that NNLS naturally enforces sparsity, as the abundancies of several spectra are set to zero: this intuitively well agrees with the characteristics of a hyperspectral pixel, which is usually composed by a limited number of materials [18]. In recent years the fully-constrained least squares (FCLS) method, which enforces not only non-negativity but also the sum-to-one constraint on the estimated abundances, has been debated by the community and is therefore not considered in these experiments [13]; furthermore, FCLS would force to 0 the residual vector $r$ in (1), affecting the noise removal procedure.

The results for UBD using NNLS and reconstructing the image as in Eq. (3) are reported in Figs. 2(b) and 3(b) for the bands at $380 \mathrm{~nm}$ and $750 \mathrm{~nm}$, respectively. Both bands, characterized by a different SNR, exhibit high visual quality, while spectral distortions appear negligible. The Normalized RMSE (NRMSE), expressed in percentage, is around 15\% and $1.7 \%$ for these two bands, and in the second case it drops down to $0.9 \%$ in the area for which ground truth was available, and from which the reference spectra were selected (ref. Table I). A magnification of a high-pass filtered version of the images show clean and smooth areas, suggesting that such distortions are mostly related to the noise which has been removed. If Non-Constrained Least Squares (NCLS) unmixing is used instead of NNLS results are much degraded, as the physical assumptions made are no longer valid: see Fig. 2(f), which is still severely affected by noise and presents some very distorted areas. As an example, no reference spectrum has been selected for the small pond of water on the upperleft side of the image, which exhibits abnormally high values.

It is of interest to make some comparison to the same image reconstructed using a subset of $k$ MNF features. The problem of selecting the optimal number $k$ affects most algorithms, as generally the representation of noise and relevant information in the MNF components presents a certain overlap, and the boundary between these is hard to locate automatically [9]. In literature this is usually set with an empirical threshold based on the eigenvalues related to the features, on a visual inspection, or on quality indicators for analysis carried out in the MNF parameter space, such as classification accuracy. A procedure is proposed in [9] to estimate the optimal value for $k$ by taking into account the dark current measured by the images acquired by a hyperspectral sensor with a closed shutter, and validate the results comparing the reconstructed images to some ideally "noise-free" images. An interesting aspect in this work is the creation of these synthetic noisefree images, which present some similarity with UBD, as they are achieved by applying NCLS unmixing using as input a spectral library and an unsupervised classification of an input reflectance image. Nevertheless, as this method uses external libraries containing spectra acquired in diverse conditions, it cannot be applied to reliably remove noise from a real image; in addition, the advantages of using NNLS over NCLS have just been discussed.

In these experiments we choose to select the number of MNF feature $k$ empirically, in order to match the RMSE obtained with UBD, keeping in mind that RMSE is expected to decay as $k$ grows. As no value of $k$ yields a minimization both of the noise and of the spectral distortion across all the bands with varying SNR, we reconstruct the image with a varying number of MNF features. We match RMSE for the bands at $380 \mathrm{~nm}$ and $750 \mathrm{~nm}$, for the whole band and in the area where ground truth is available (applying the mask in Fig. 3(f)). The resulting values for $k$ are 4, 7 and 30, respectively. All results are reported in Figs. 2 and 3 and in Table I. It is clear that the noise in the band at $380 \mathrm{~nm}$ increases with the number of MNF features used in the back rotation. On the other hand, the noise in the band at $750 \mathrm{~nm}$ decreases, but no reconstruction can provide an image as clean as the result of UBD, as noise starts already to affect the image with a reconstruction using few MNF components (namely 4). It may be argued that keeping more MNF features would reduce distortions, but it can be seen that increasing $k$ in the back rotation severely affects the denoising of bands with low SNR (see Fig. 2(e)).

An interesting consideration to be done on this comparison is that the visual image quality is much higher for UBD, for comparable values of RMSE. As an example, results obtained with MNF in Fig.2(c) present severe spectral distortions in comparison to UBD, while images in Fig. 3(d) and Fig. 3(e) are more noisy. In addition, some spectral information in Fig. 3(d) is visibly corrupted: for example, the bright fields in the center of the scene appear darker than in the original image in Fig. 3(a) (the histogram stretch is the same in all the images). Please note that RMSE is used in these experiments only as an empirical indicator to select a meaningful value for $k$, not as an evaluation metric. Indeed, our results suggest that RMSE could be a poor objective criterion to assess the image quality of remotely sensed data, as it is already been found to be for monochromatic and colour pictures [11].

\section{B. Indian Pines dataset}

A second experiment is carried out on the popular AVIRIS Indian Pines dataset, of size $145 \times 145$ and containing 224 bands. One reference spectrum has been collected from an 


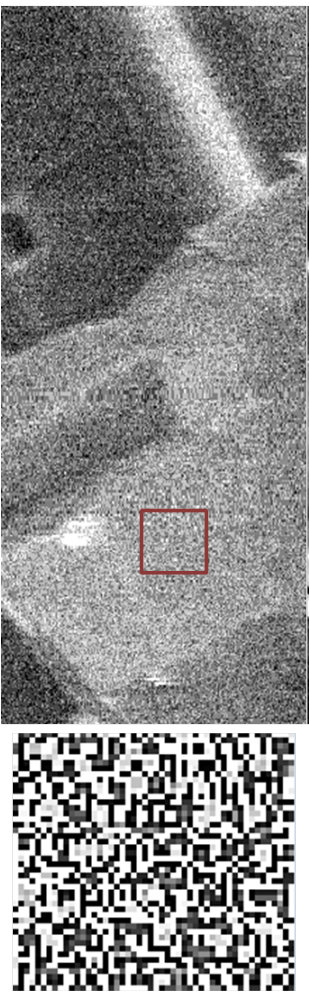

(a)

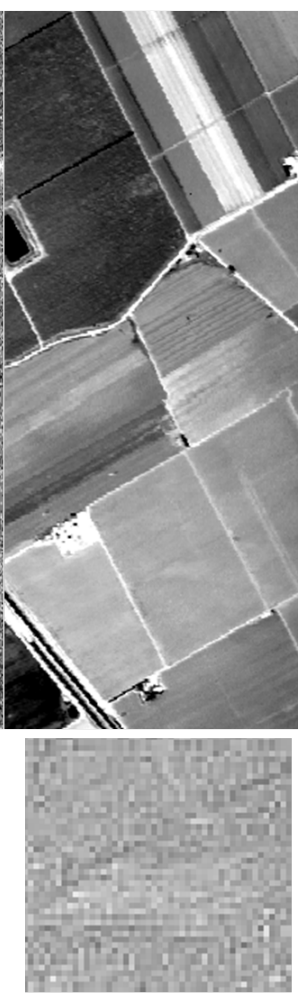

(b)
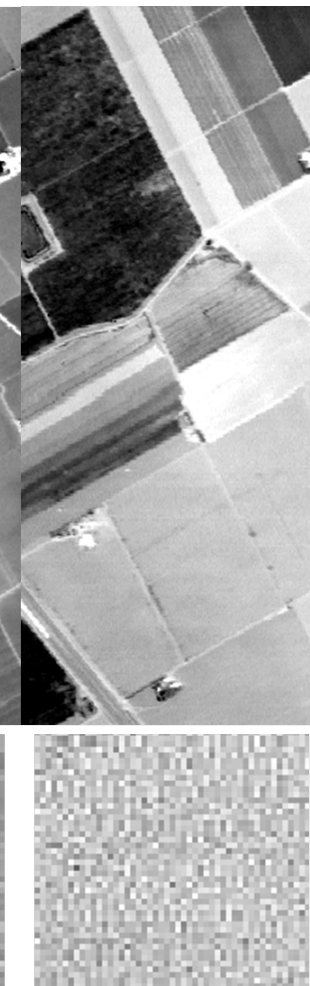

(c)

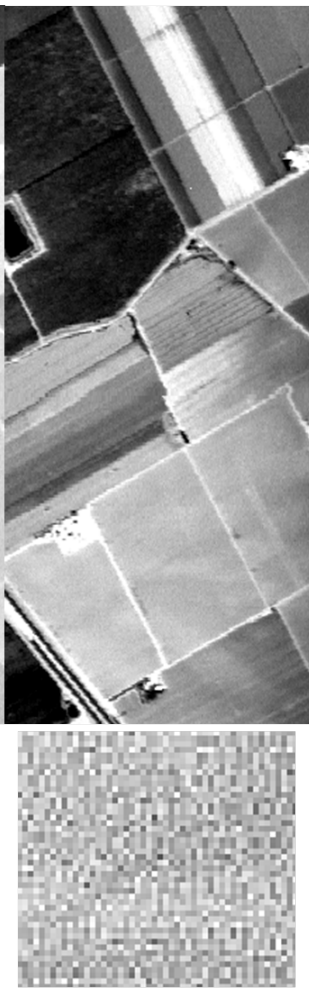

(d)
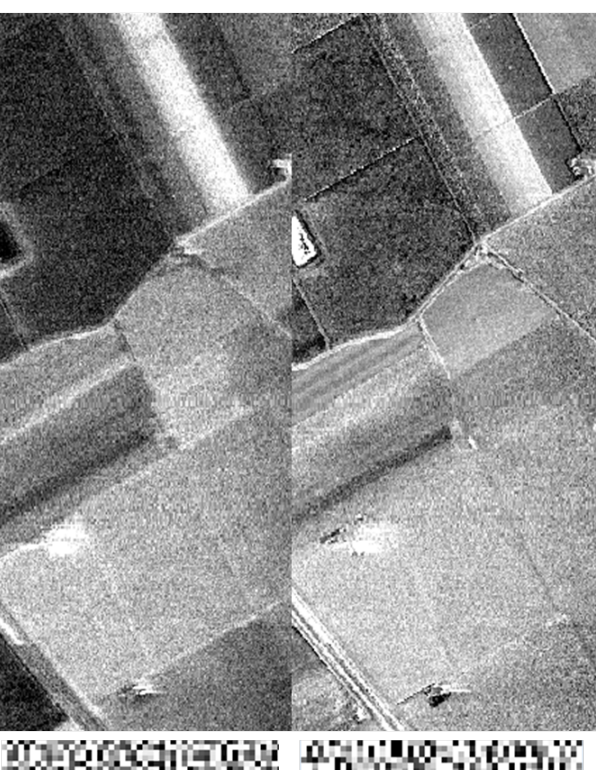

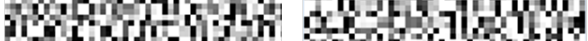

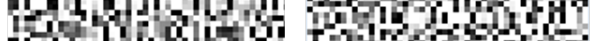

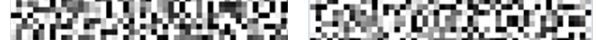

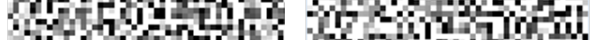

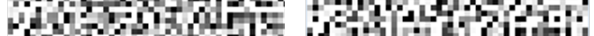

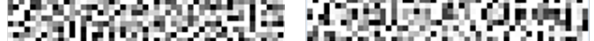

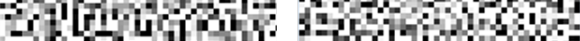

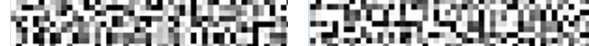

(e)

(f)

Fig. 2. Band at $380 \mathrm{~nm}$. From left to right: (a) original image; (b) reconstruction obtained with UBD using NNLS unmixing; (c-e) reconstructed images using 4, 7 and $30 \mathrm{MNF}$ components respectively; (f) reconstruction obtained with UBD using NCLS unmixing. Below: magnified areas represented by the square in subfigure (a), after a high-pass filtering of the image to make the noise more evident.

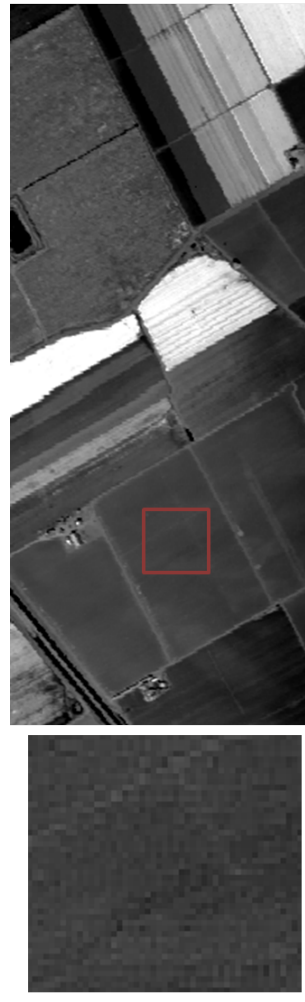

(a)

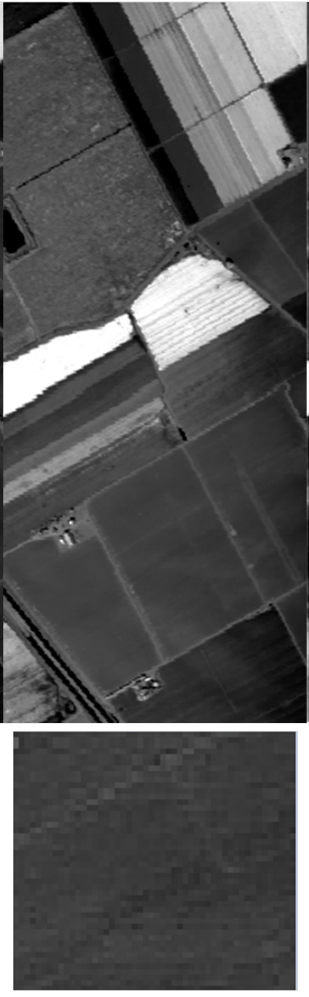

(b)

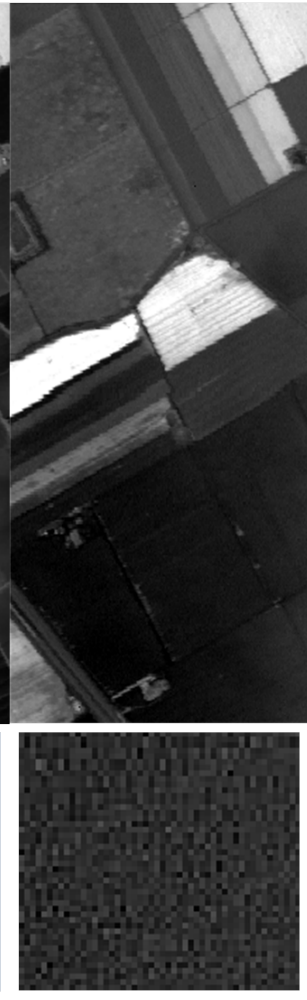

(c)

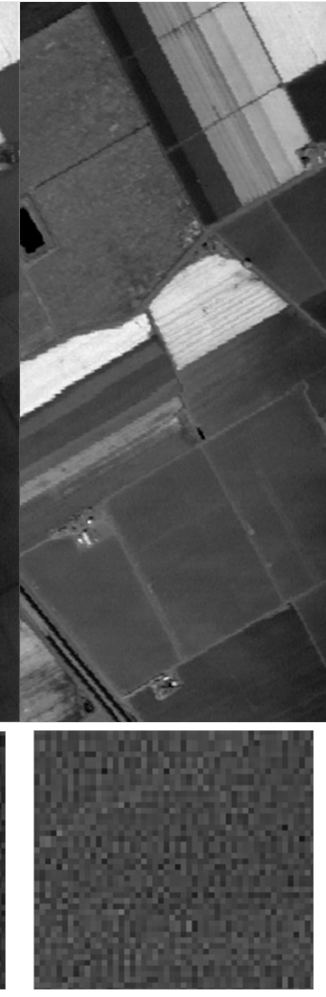

(d)

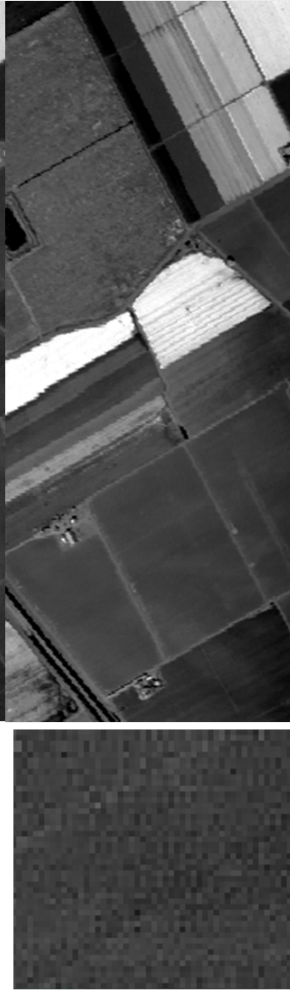

(e)
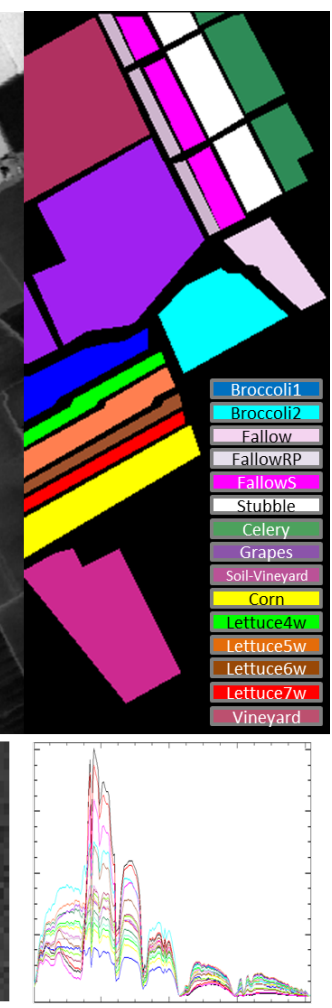

(f)

Fig. 3. Band at $750 \mathrm{~nm}$. From left to right, top: (a) original image; (b) reconstruction obtained with UBD using NNLS unmixing; (c-e) reconstructed images using 4, 7 and $30 \mathrm{MNF}$ components respectively; (f) areas in the image where ground truth is available. Bottom: (a-e) magnified areas represented by the square in subfigure (a), after a high-pass filtering of the image to make the noise more evident; (f) Reference spectra collected from the ground truth. 
TABLE I

ERRORS FOR RECONSTRUCTION USING UBD AND DIFFERENT NUMBER OF FEATURES FOR MNF. THE MASKED AREA IS REPORTED IN FIG. 3(F). THE COLOURED FIELDS SHOW THE NUMBER OF MNF COMPONENTS $k$ CHOSEN BY MATCHING THE ERROR FOR UBD WITH DIFFERENT CRITERIA.

\begin{tabular}{|c|c|c|c|c|c|c|c|c|}
\hline & \multicolumn{4}{|c|}{$\mathrm{B} 1(380 \mathrm{~nm})$} & \multicolumn{4}{|c|}{$\mathrm{B} 2(750 \mathrm{~nm})$} \\
\hline Error & RMSE & NRMSE & RMSE (masked) & NRMSE(masked) & RMSE & NRMSE & RMSE (masked) & NRMSE(masked) \\
\hline UBD & 56.02 & $15.06 \%$ & 47.42 & $13.1 \%$ & 60.79 & $1.77 \%$ & 30.55 & $0.80 \%$ \\
\hline $\mathrm{MNF}, k=4$ & 55.94 & $15.04 \%$ & 56.74 & $15.67 \%$ & 654.1 & $19.07 \%$ & 613.92 & $16.07 \%$ \\
\hline MNF, $k=7$ & 48.81 & $13.12 \%$ & 47.31 & $13.07 \%$ & 175.52 & $5.12 \%$ & 135.44 & $3.55 \%$ \\
\hline $\mathrm{MNF}, k=30$ & 39.73 & $10.68 \%$ & 29.85 & $10.88 \%$ & 31.18 & $0.91 \%$ & 29.85 & $0.78 \%$ \\
\hline
\end{tabular}

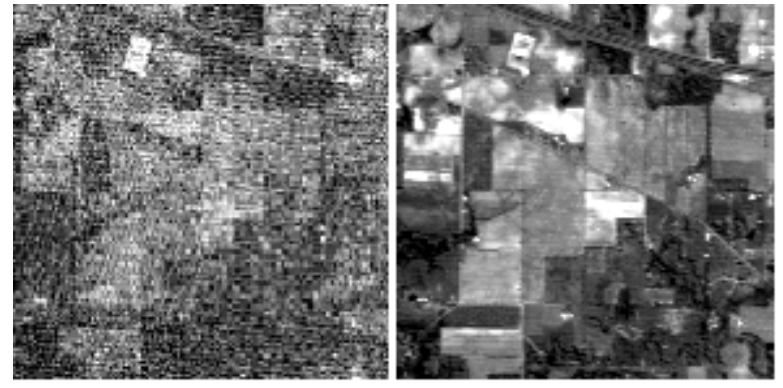

Fig. 4. On the left: single band centered at $370 \mathrm{~nm}$ from the Indian Pines dataset. On the right: UBD results.

averaged area of $5 \times 5$ pixels for each of the available 15 classes in the groundtruth image. We applied UBD to the full dataset and report in Fig. 4 the results for the first band of the dataset. The denoised image has a NRMSE of 3.6\%, with a mean NRMSE value across all bands of $1.6 \%$.

\section{CONCLUSIONS}

Spectral unmixing and denoising algorithms for hyperspectral remote sensing have always been considered independently. In this paper we propose an Unmixing-based Denoising technique (UBD) which differs from classical denoising methods, being based on the physical rather than the statistical properties of the components of a given spectrum: while traditional algorithms are based on second order statistics, UBD reconstructs a single spectrum or image element by a linear combination of spectra which are noise-free, as the value of a reference spectrum in a given band is averaged over a homogeneous area. This allows reconstructing bands with low SNR with a certain degree of reliability and represents a viable solution for the recovery of "junk" bands, which are usually discarded in practical applications.

A drawback of the method is that it requires either prior knowledge of the scene, or a reliable identification of the classes of interest in the image. Furthermore, it needs a reasonably large number of pixels for each reference spectrum in order to have a meaningful mean value robust to noise and local variations. The reference spectra selection step may be anyway replaced in the future by a robust endmember extraction algorithm such as SSEE [19], which also reduces the noise influences by averaging spectra which are both spectrally similar and spatially close.

The proposed algorithm does not use any post-processing through morphological filtering, or in general methods which make the final result appear smoother, as it operates pixelwise and may then preserve important details in each image element, if these can be described by a linear combination of the spectra related to the classes of interest. Experimental results show that UBD provides stable results across all bands, as it automatically intervenes more heavily on bands with low SNR, keeping the informational content of bands with high SNR mostly unaltered. The distortions introduced in informationrich bands is negligible, and mostly related to the noise which is removed, as this is part of the residual vector which is discarded when reconstructing the image. This constitutes an advantage over traditional denoising methods such as MNF, as the optimal number of components to be used in the image reconstruction step for this algorithm is not constant across spectral bands with different SNR.

Another interesting aspect is that MNF, likewise several denoising algorithms for hyperspectral images, is driven by a minimization of the Mean Squared Error (MSE), which has been criticized in the literature due to its poor performance as a visual quality estimator. Our experiments confirm that such criterion on its own is not robust enough to evaluate qualitatively denoising and image reconstruction algorithms for hyperspectral data, both from the informational content and from the perceived visual quality points of view. This may trigger interesting discussions, as usual validation techniques used in remote sensing can be questioned: would it make sense to adopt a quality index which takes into account higher order statistics? In [20] the authors expand the Structural Similarity Index (SSIM) [11] from the 2D case to applications to hyperspectral images, but they do so by simply computing the average value of SSIM across all the spectral bands. The reported experimental results suggest that in the future a similar index, comprising an accurate prediction both for spatial and spectral distortion in hyperspectral images, would represent a valuable contribution to improve the validation of data compression, denoising and sparse reconstruction algorithms for this kind of data.

\section{REFERENCES}

[1] C. Bruce, "Hyspiri mission concept overview and recent ice and trl activities," in 2012 HyspIRI Science Workshop, October 2012.

[2] B. Aiazzi, L. Alparone, A. Barducci, S. Baronti, P. Marcoionni, I. Pippi, and M. Selva, "Noise modelling and estimation of hyperspectral data from airborne imaging spectrometers," Annals of Geophysics, vol. 49, no. $1,2006$.

[3] T. Kutser, D. C. Pierson, K. Y. Kallio, A. Reinart, and S. Sobek, "Mapping lake cdom by satellite remote sensing," Remote Sensing of Environment, vol. 94, no. 4, pp. 535 - 540, 2005.

[4] S. C. Johannessen, W. L. Miller, and J. J. Cullen, "Calculation of uv attenuation and colored dissolved organic matter absorption spectra from measurements of ocean color," Journal of Geophysical Research, vol. 108, no. C9, pp. 1701 - 1713, 2003. 
[5] R. C. Gonzalez and R. E. Woods, Digital Image Processing (3rd Edition), 3rd ed. Prentice Hall, Aug. 2007.

[6] N. Renard, S. Bourennane, and J. Blanc-Talon, "Denoising and dimensionality reduction using multilinear tools for hyperspectral images," IEEE Geoscience and Remote Sensing Letters, vol. 5, no. 2, pp. 138 -142 , april 2008.

[7] G. Chen and S.-E. Qian, "Denoising of hyperspectral imagery using principal component analysis and wavelet shrinkage," IEEE Transaction on Geoscience and Remote Sensing, vol. 49, no. 3, pp. 973-980, 2011.

[8] A. A. Green, M. Berman, P. Switzer, and M. D. Craig, "A transformation for ordering multispectral data in terms of image quality with implications for noise removal," IEEE Transactions on Geoscience and Remote Sensing, vol. 26, no. 1, pp. 65-74, Jan. 1988.

[9] U. Amato, R. M. Cavalli, A. Palombo, S. Pignatti, and F. Santini, "Experimental approach to the selection of the components in the minimum noise fraction," IEEE Transactions on Geoscience and Remote Sensing, vol. 47, no. 1, pp. 153-160, 2009.

[10] M. Farzam and S. Beheshti, "Simultaneous denoising and intrinsic order selection in hyperspectral imaging," IEEE Transactions on Geoscience and Remote Sensing, vol. 49, no. 9, pp. 3423 -3436, sept. 2011.

[11] Z. Wang, A. C. Bovik, H. R. Sheikh, and E. P. Simoncelli, "Image quality assessment: From error visibility to structural similarity," IEEE Transactions on Image Processing, vol. 13, no. 4, pp. 600-612, 2004.

[12] J. M. P. Nascimento and J. M. B. Dias, "Does independent component analysis play a role in unmixing hyperspectral data?" IEEE Transactions on Geoscience and Remote Sensing, vol. 43, no. 1, pp. 175-187, 2005.

[13] J. M. Bioucas-Dias, A. Plaza, N. Dobigeon, M. Parente, Q. Du, P. Gader, and J. Chanussot, "Hyperspectral unmixing overview: Geometrical, statistical, and sparse regression-based approaches," IEEE Journal of Selected Topics in Applied Earth Observations and Remote Sensing, vol. 5, no. 2, pp. 354-379, 2012

[14] D. Cerra, R. Mueller, and P. Reinartz, "A Classification Algorithm for Hyperspectral Images based on Synergetics Theory," IEEE Transactions on Geoscience and Remote Sensing, no. in press.

[15] I. Dopido, M. Zortea, A. Villa, A. Plaza, and P. Gamba, "Unmixing prior to supervised classification of remotely sensed hyperspectral images," Geoscience and Remote Sensing Letters, IEEE, vol. 8, no. 4, pp. 760764, 2011.

[16] G. Martín and A. Plaza, "Spatial-spectral preprocessing prior to endmember identification and unmixing of remotely sensed hyperspectral data," Selected Topics in Applied Earth Observations and Remote Sensing, IEEE Journal of, vol. 5, no. 2, pp. 380-395, 2012.

[17] A. Plaza, P. Martinez, J. Plaza, and R. Perez, "Dimensionality reduction and classification of hyperspectral image data using sequences of extended morphological transformations," IEEE Transactions on Geoscience and Remote Sensing, vol. 43, no. 3, pp. 466-479, 2005.

[18] J. Bieniarz, R. Müller, X. Zhu, and P. Reinartz, "Sparse approximation, coherence and use of derivatives in hyperspectral unmixing," in 4th Workshop on Hyperspectral Image and Signal Processing: Evolution in Remote Sensing WHISPERS 2012, June 2012.

[19] D. Rogge, B. Rivard, J. Zhang, A. Sanchez, J. Harris, and J. Feng, "Integration of spatial-spectral information for the improved extraction of endmembers," Remote Sensing of Environment, vol. 110, no. 3, pp. 287-303, 2007.

[20] Y. Zhao, J. Yang, Q. Zhang, L. Song, Y. Cheng, and Q. Pan, "Hyperspectral imagery super-resolution by sparse representation and spectral regularization," EURASIP Journal on Advances in Signal Processing, vol. 2011, no. 1, p. 87, 2011. 